\title{
Are analytical standards and reagents really reliable?
}

\author{
Céline Gautier*a ${ }^{\mathrm{a}}$, Perrine Wund ${ }^{\mathrm{b}}$, Elodie Laporte ${ }^{\mathrm{a}}$, Jérôme Vial ${ }^{\mathrm{c}}$, Didier Thiébaut ${ }^{\mathrm{c}}$, Cédric Rivier ${ }^{\mathrm{d}}$, Ma- \\ rielle Crozet $^{\mathrm{e}}$, Florence Goutelard ${ }^{\mathrm{f}}$
}

${ }^{a}$ CEA/DEN/DANS/DPC/SEARS/LASE, Atomic Energy Commission, CEA Saclay, PC 171, 91191 Gif-sur-Yvette, France

${ }^{\mathrm{b}}$ IFP Energies Nouvelles, Physics and Analysis Division, 1\&4 avenue de Bois-Préau, 92852 Rueil-Malmaison Cedex, France

${ }^{\mathrm{c}}$ Analytical and Bioanalytical Sciences and Miniaturization Laboratory, UMR CNRS CBI 8231, ESPCI ParisTech, PSL Research University, 75005 Paris, France

${ }^{\mathrm{d}} \mathrm{CEA} / \mathrm{DEN} / \mathrm{MAR} / \mathrm{DRCP} / \mathrm{SERA} / \mathrm{LAMM}$, Atomic Energy Commission, CEA Marcoule, Building 166, BP 17171, 30207 Bagnols-sur-Ceze, France

${ }^{e}$ CEA/DEN/MAR/DRCP/CETAMA, Atomic Energy Commission, CEA Marcoule, Building 400, BP 17171, 30207 Bagnolssur-Ceze, France

${ }^{\dagger}$ CEA/DEN/MAR/DEIM/Nuclab, Atomic Energy Commission, CEA Marcoule, Building 109, BP 17171, 30207 Bagnols-sur-Ceze, France

\begin{abstract}
Quality assurance is one of the major challenges in analytical chemistry whatever the scope of application. The quality of analytical standards is never questioned, however sometimes odd results are obtained and all the other potential sources of discrepancies are eliminated. So, we investigated the quality of three analytical standards and reagents implemented for radiological and chemical characterizations of nuclear waste. In particular, this work examined the purity of a source of tritiated-labelled dodecane, the trueness and the purity of a DTPA reagent and the trueness of a multi-anions standard used for an intercomparison exercise. It was shown that the source of tritiated-labelled dodecane contains $60 \%$ of tritiated-labelled impurities. The trueness of the DTPA concentration was questioned due to the presence of impurities in the solution. It was proven that the long-term stability of the multi-anions standard was not guaranteed for nitrite. The results clearly demonstrated that, although in opposition to intuition, cautiousness has to be taken towards the quality of the analytical standards.
\end{abstract}

The issue of quality assurance (QA) in the analytical chemistry laboratory has become of great importance for many years. ${ }^{1-4}$ Quality control (QC) and QA are particularly essential for pharmaceutical sciences ${ }^{5}$, environmental monitoring ${ }^{6}$ but also for nuclear industry ${ }^{7}$. Radioactive waste management is a challenging task faced by nuclear power countries and is a prime concern for the public and therefore for analytical laboratories. In France, the National Radioactive Waste Management Agency (ANDRA) is in charge of the management of radioactive waste. It requests radiological and chemical characterizations of nuclear waste and specifies acceptance criteria for packages that have to be respected. ${ }^{8}$

Consequently, analytical laboratories devoted to nuclear waste characterizations must implement validated methods and provide accurate and reliable measurements. ${ }^{7}$ All the

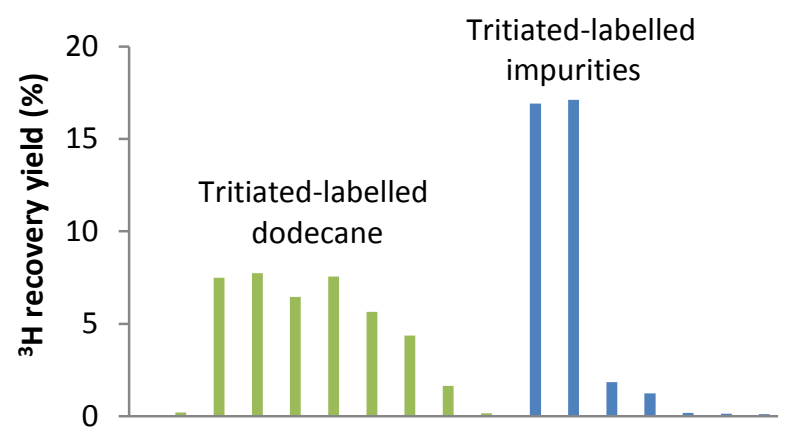

radiological and chemical analyses require the implementation of different laboratory instruments, such as liquid chromatography systems (High-Performance Liquid Chromatography coupled to Mass Spectrometry HPLC-MS, Ion Chromatography IC) or Liquid Scintillation Counters (LSC). Those instruments must be calibrated with reference materials and standards of known compositions. Calibration standards and reagents must be reliable in terms of accuracy, purity and long-term stability. ${ }^{1-4,9}$

Among the list of nuclear waste characterizations to be investigated, many radionuclides such as tritium have to be quantified. ${ }^{8}$ As a pure beta-emitter with a low energy $($ Emax $=$ $18.6 \mathrm{keV})$, tritium is favorably measured by LSC. ${ }^{10}$ The liquid scintillation analyser is generally calibrated using tritiated water standards. For nuclear waste and effluent samples, triti- 
um is usually extracted from the matrices and isolated from the potential interfering radionuclides using a combustion/pyrolysis step prior to LSC. ${ }^{10,11}$ The optimization and the validation of the combustion conditions also require the use of tritiated water standards. ${ }^{11}$ For the analysis of radioactive materials such as organic solvents and oils, it is necessary to implement tritiated-labelled organic compounds as reference molecules. ${ }^{11}$ In the nuclear fuel reprocessing cycle, liquidliquid extraction processes generally involve dodecane as solvent. Consequently, for tritium determination in radioactive waste after a combustion step, it is crucial to have access to a reliable and pure source of tritiated-labelled dodecane.

Moreover, in the framework of nuclear waste management, waste producers are requested to quantify organic ligands like EDTA (ethylenediaminetetraacetic acid) and DTPA (diethylenetriaminepentaacetic acid). Actually, polycarboxylate molecules have been widely used in the nuclear industry for decontamination purposes. Hence, they may be present in effluents and waste samples. ${ }^{12}$ They may form complexes with radionuclides, which can facilitate their mobility and as a consequence their potential leaching in the environment. They can be analyzed by high performance liquid or ion chromatography ${ }^{13}$ coupled with conductimetric ${ }^{14}$ or mass spectrometry ${ }^{15-17}$ detection. Our group previously reported the quantification of EDTA $^{17}$ in radioactive effluents by applying a HPLC-MS method. For all chromatographic separations ${ }^{13-17}$, it is necessary to establish calibration curves based on standards to quantify the analytes of interest.

The chemical composition in terms of inorganic anions has also to be characterized in the radioactive effluents. ${ }^{8}$ Among the inorganic anions to be analyzed, chloride, fluoride, sulfate, nitrate and nitrite must be quantified because of their complexing and corrosive properties. ${ }^{8}$ Common inorganic anions are widely determined by IC coupled with conductimetric detection. ${ }^{18-20}$ Actually, this technique has become a reference method for the measurement of inorganic ions in water and wastewater ${ }^{20}$, including radioactive effluents $^{21}$. The anions quantification relies on calibration curves obtained from the analyses of IC standards.

Recently, Wahl et al. ${ }^{22}$ investigated solvent purity using comprehensive 2D gas chromatography. They highlighted the presence of impurities in common organic solvents such as commercial acetone brands. This work pointed out that cautiousness must be taken towards the purity of solvents used for trace analysis.

In the present study, we investigated the reliability of three standards and reagents implemented for radiological and chemical characterizations of nuclear waste. In particular, this work examined the purity of a source of tritiated-labelled dodecane, the trueness and the purity of a DTPA reagent and the trueness of a multi-anions standard used for an intercomparison exercise.

\section{MATERIALS AND METHODS}

All reagents (nitric acid) and solvents (hexane, ethyl acetate, ethanol) used were of analytical grade.

For the radiological analysis of tritium, a source of tritiatedlabelled dodecane was purchased from a US provider. According to the technical data sheet, its specific activity was specified at $40 \mathrm{kBq} / \mathrm{g}$ (no uncertainty was given) whereas the radiochemical and chemical purity was specified at $99 \%$ from ThinLayer Chromatography (TLC). The recommended storage temperature was $0-5^{\circ} \mathrm{C}$. It was noted that no expiration date was indicated. The chromatographic separations were carried out on pre-packed Finisterre ${ }^{\mathrm{TM}} \mathrm{Si}$ SPE cartridges (Teknokroma, Spain) containing $1 \mathrm{~g}$ of silica with average particle size of $50 \mu \mathrm{m}$. The dead volume of the cartridge was around 1.2 $\mathrm{mL}$. The Si SPE cartridges were conditioned with $12 \mathrm{~mL}$ hexane. All fractions obtained after separation were collected to quantify tritium. All tritium measurements were performed with a Tri-Carb liquid scintillation counter (Perkin-Elmer, France). The instrument was calibrated for tritium analysis using a certified tritiated water standard (CERCA LEA, France). The accuracy of the tritium analyses was checked annually with proficiency tests organized by the LNHB laboratory (Laboratoire National Henri Becquerel, France). All organic samples were diluted in $5 \mathrm{~mL}$ ethanol and then mixed with $10 \mathrm{~mL}$ Ultima Gold ${ }^{\mathrm{TM}}$ LLT scintillation cocktail in 20 $\mathrm{mL}$ polyethylene vials. It was checked that the organic molecules of the samples did not induce any quenching effects.

The DTPA solution was purchased as a General Purpose Reagent (GPR) from an international supplier. This chemical product was a DTPA pentasodium solution in water which should have a purity grade higher than $95.0 \%$. According to the certificate of analysis, the $\mathrm{pH}$ of the solution was certified at 11.7 and the concentration was certified at $1.02 \mathrm{M}$ (for a GPR, no uncertainty is given). The experimental $\mathrm{pH}$ was measured with a SevenMulti ${ }^{\mathrm{TM}} \mathrm{pH}$-meter (Metrohm, Villebon sur Yvette) which was calibrated with certified $\mathrm{pH}$ buffers.

For IC experiments, single- and multi-anions standards were purchased as IC Certified Reference Material (CRM) from a US accredited supplier. The interlaboratory exercise called "EQRAIN Ions 2014" was performed using a CRM solution. The nitrite concentration was certified at $0.330(+/-0.016)$ $\mathrm{mg} / \mathrm{L}$ for six months. All IC standards except the interlaboratory CRM solution were diluted prior to injection in ultra-pure water (resistivity $18.2 \mathrm{M} \Omega \mathrm{cm}$ ) obtained from a Milli-Q purification system (Millipore, France). The IC separations were conducted using an ICS-4000 capillary-scale instrument (Thermo Scientific, USA). The capillary-scale system consisted of an AS-AP autosampler, a capillary single pump, an EG online eluent generator module, a conductivity detector (which was thermally controlled at $15^{\circ} \mathrm{C}$ ) and an IC Cube. The latter integrated a capillary-scale in-line eluent degasser, a separately controlled capillary column oven (which temperature was fixed at $30^{\circ} \mathrm{C}$ ), a capillary 4-port injection valve fitted with a $0.4 \mu \mathrm{L}$ internal loop and an anion capillary eluent suppressor (ACES). The ICS-4000 system was equipped with capillary AG15/AS15 columns (Thermo Scientific, USA) working at $0.012 \mathrm{~mL} / \mathrm{min}$ with $38 \mathrm{mM} \mathrm{KOH}$ eluent.

\section{RESULTS AND DISCUSSION}

Source of tritiated-labelled dodecane. For the determination of tritium in nuclear waste, it is requested to perform a pyrolysis/combustion prior to LSC. ${ }^{10,11}$ To calibrate our method, a commercialized source of tritiated-labelled dodecane was chosen as an organic reference molecule of nuclear processes. Before using it as a quality control standard, its purity was examined. For that purpose, liquid chromatography with gravitational elution was implemented. Due to the non-polar property of dodecane, normal-phase chromatography based on silica as stationary phase was selected. ${ }^{23}$ In those chromatographic conditions, dodecane must be quantitatively eluted with a non-polar solvent (such as hexane), whereas polar compounds must be eluted with a polar solvent (such as acetyl acetate). ${ }^{23}$ After the loadings of the titriated source, the Si SPE 
cartridge was successively flushed with hexane and acetyl acetate. Figure 1 shows the elution diagram of tritium contained in the tritiated-labelled dodecane source. Two elution peaks were respectively detected in hexane and acetyl acetate fractions, which was not consistent with the presence of a single molecule in the source. The first peak should correspond to the elution of tritiated-labelled dodecane. The second peak may be related to more polar tritiated-labelled impurities contained in the source whereas the source purity was specified as $99 \%$. Besides, both elution peaks corresponded to $40 \%$ of tritium activity: the total loss of tritium activity was thus quantified at $20 \%$. To assess the presence of impurities, the fractions corresponding to the first elution peak were gathered and a second chromatographic separation was carried out with this purified tritiated-labelled dodecane solution. The corresponding elution diagram is depicted in Figure 2. Only one elution peak was observed and tritium activity was quantitatively recovered in hexane fractions. As a consequence, the commercialized source of tritiated-labelled dodecane contains $60 \%$ of tritiated-labelled impurities. The TLC procedure applied by the supplier should not be sufficiently selective to purify the synthesized product, or the long-term stability of the tritiated molecule is not enough reliable.

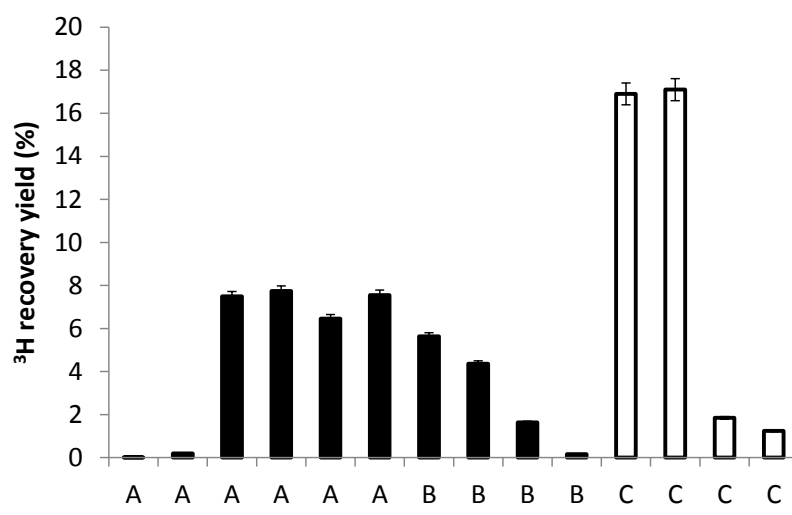

Figure 1. Elution diagram of the non-purified tritiated dodecane source (each A corresponds to the flushing of $1 \mathrm{~mL}$ non-purified tritiated dodecane source, each $\mathrm{B}$ corresponds to the flushing of 2 $\mathrm{mL}$ hexane and each $\mathrm{C}$ corresponds to the flushing of $2 \mathrm{~mL}$ ethyl acetate).

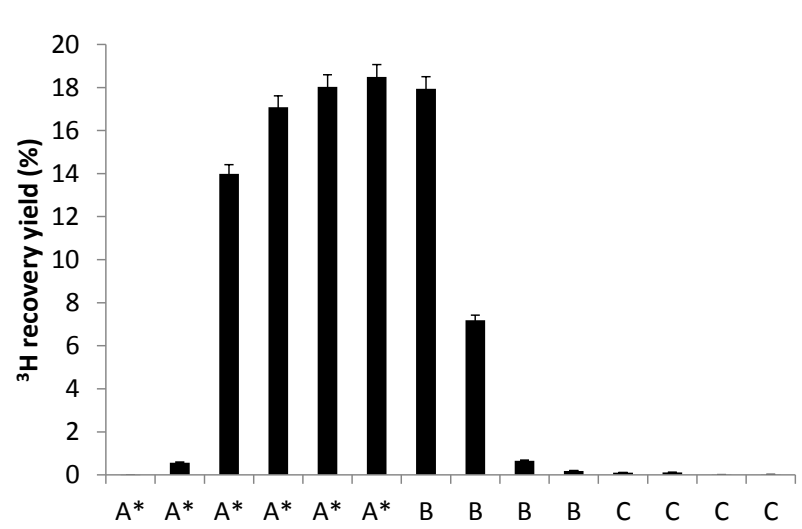

Figure 2. Elution diagram of the purified tritiated-labelled dodecane source (each $\mathrm{A}^{*}$ corresponds to the flushing of $1 \mathrm{~mL}$ purified tritiated dodecane source, each $\mathrm{B}$ corresponds to the flushing of $2 \mathrm{~mL}$ hexane and each $\mathrm{C}$ corresponds to the flushing of $2 \mathrm{~mL}$ ethyl acetate).
General Purpose Reagent (GPR) of DTPA. We previously developed a method to quantify EDTA in radioactive effluents by HPLC-MS. ${ }^{17}$ It was recently proven that the same procedure can be implemented for the analysis of DTPA. ${ }^{24}$ It can be noted that no commercialized CRM or analytical standard is available for DTPA. As for EDTA, a calibration curve was established for DTPA using a commercialized salt dissolved in diluted ammonia. A GPR of DTPA was purchased to be used as a quality control solution. It was decided to control its certified $\mathrm{pH}$ and concentration. DTPA (denoted here as H5DTP) has five acidic functions: the corresponding $\mathrm{pKa}$ values are: $\mathrm{pKa} 1=2, \mathrm{pKa} 2=2.6, \mathrm{pKa} 3=4.3, \mathrm{pKa} 4=8.6$ and $\mathrm{pKa} 5=10.5{ }^{25}$ Prior to the experiments, the theoretical speciation of DTPA in water was modelled using JChess software (Ecole des Mines ParisTech, France). For that purpose, the database of the software (chess.tdb) was enriched with specific stability constants for DTPA obtained from Smith and Martell. ${ }^{25}$ Figure 3 shows the theoretical titration curve of the DTPA solution by $5 \mathrm{M} \mathrm{HNO}_{3}$ (triangles). The initial $\mathrm{pH}$ of the solution should be 11.7, as specified in the supplier certificate. Due to the closeness of the 5 pKas, only one equivalence point should be expected during the titration, which corresponds to the conversion of $\mathrm{DTP}^{5-}$ into $\mathrm{H}_{2} \mathrm{DTP}^{3-}$. To determine the DTPA concentration, $50 \mathrm{~mL}$ of the solution was experimentally titrated using $5 \mathrm{M} \mathrm{HNO}_{3}$. The result of this experimental titration is presented in Figure 3 (squares). The initial experimental $\mathrm{pH}$ of the solution was found to be higher than 14 . Furthermore, the titration curve exhibited two equivalence points. Those experimental results were not in agreement with the theoretical simulations. To explain those differences, the presence of impurities can be assumed, such as $\mathrm{NaOH}$ impurities originating from DTPA synthesis. Based on this hypothesis, the first equivalence point was associated to the reaction of $\mathrm{OH}^{-}$with $\mathrm{H}^{+}$and the second one to the conversion of $\mathrm{DTP}^{5-}$ into $\mathrm{H}_{2} \mathrm{DTP}^{3-}$, which led to respective concentrations of 0.28 $\mathrm{M}$ for $\mathrm{NaOH}$ and $0.93 \mathrm{M}$ for DTPA. The theoretical titration curve corresponding to this composition was modelled with JChess software (diamonds in Figure 3). The theoretical titration curve with $\mathrm{NaOH}$ impurities fits perfectly the experimental curve. Consequently, the DTPA solution contains impurities which induces a bias of $9 \%$ for DTPA concentration.

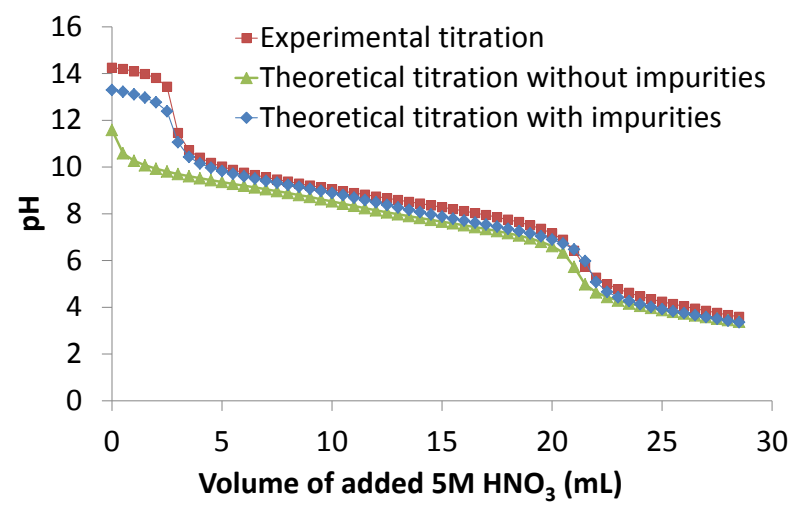

Figure 3. Experimental and theoretical titrations of the DTPA solution.

Multi-anions standard with $\mathrm{NO}_{2}^{-}$. Our group recently tested the new technology of capillary-scale IC systems for the measurements of common inorganic anions in radioactive effluents. $^{26}$ To evaluate the analytical performance of this 
novel instrument, our group participated to an intercomparison exercise called "EQRAIN Ions" organized in 2014 by the CETAMA committee ${ }^{27}$. To quantify the different anions, calibration curves were established from a multi-anions CRM standard containing $\mathrm{F}, \mathrm{Cl}^{-}, \mathrm{NO}_{3}{ }^{-}, \mathrm{SO}_{4}{ }^{2-}, \mathrm{PO}_{4}{ }^{3-}$ and mono- $\mathrm{NO}_{2}{ }^{-}$ and $\mathrm{Br}^{-} \mathrm{CRM}$ standards. For all the anions analyzed in the "EQRAIN Ions" solution, the absolute values of $\mathrm{z}$-scores and zeta-scores were lower than 2: the laboratory performance was considered as satisfactory, except for $\mathrm{NO}_{2}^{-}$. Figure 4 was elaborated for $\mathrm{NO}_{2}^{-}$from the results of the interlaboratory comparison exercise. It can be highlighted that the robust mean value of the intercomparison exercise $((0.243+/-0.038) \mathrm{mg} / \mathrm{L})$ differs significantly from the assigned value $((0.330+/-0.016)$ $\mathrm{mg} / \mathrm{L}$ ) for $\mathrm{NO}_{2}^{-}$. The difference of $26 \%$ between the robust value and the assigned value can be attributed to the instability of $\mathrm{NO}_{2}{ }^{-}$due to its conversion to $\mathrm{NO}_{3}{ }^{-}$at long term. Actually, $\mathrm{NO}_{2}{ }^{-}$is known to be quite unstable, even in reagent-grade water, especially at low concentrations. ${ }^{28,29}$ The results of the supplier against analysis show also an abnormal drift of the $\mathrm{NO}_{2}{ }^{-}$concentration in the solution. Therefore, the hypothesis of an erroneous reference value was confirmed. There is no guarantee that this drift is the same in each of the bottles sent to laboratories. The supplier recognizes that the long-term preservation of 6 months was unsuitable for $\mathrm{NO}_{2}{ }^{-}$in this concentration range. So, cautiousness should be taken towards to the long-term stability of the analytical standards, otherwise the end results can be biased. For future EQRAIN ions comparisons, $\mathrm{NO}_{2}^{-}$should be separated from the other anions to ensure its stability at low concentrations.

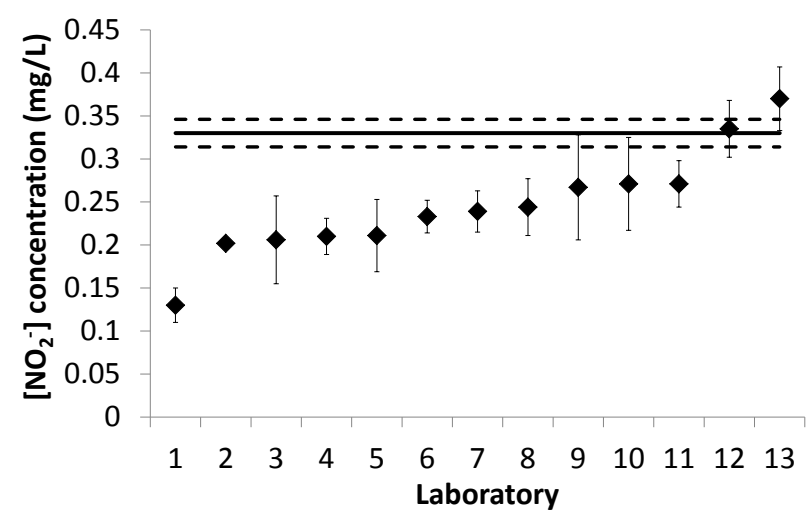

Figure 4. Graph derived from the results of the interlaboratory exercise "EQRAIN Ions 2014" obtained for $\mathrm{NO}_{2}{ }^{-}$concentration (assigned concentration in bold line, limits of the uncertainties associated to the assigned concentration in dotted lines, black diamonds correspond to the results of the 13 laboratories participating to the interlaboratory exercise).

\section{CONCLUSION}

The reliability of the analytical standards and reagents is of prime importance. Their purity and trueness are a key determinant for the validity of the calibration procedures, and therefore for the quality of the end results. In this work, the reliability of three standards and chemical products was clearly questioned in terms of purity and trueness. This study obviously demonstrated that cautiousness has to be taken towards the quality of analytical standards. Unlike what is commonly admitted, analytical standards can also be affected by errors.

\section{Corresponding Author}

*E-mail: celine.gautier@cea.fr

\section{Author Contributions}

The manuscript was written through contributions of all authors. All authors have given approval to the final version of the manuscript.

\section{REFERENCES}

(1) Taylor, J. K. Anal. Chem. 1981, 53, 1588A-1596A.

(2) Nadkarni, R. A. Anal. Chem. 1993, 65, 387A-395A.

(3) Wenclawiak, B. W.; Koch, M.; Hadjicostas, E. In Quality Assurance in Analytical Chemistry, Springer, 2010.

(4) CITAC / EURACHEM In Guide to Quality in Analytical Chemistry. An Aid to Accreditation, 2002.

(5) Knaack, J. Pharmaceut. Anal. Acta 2012, 3, 1-2.

(6) Kayasth, S.; Swain, K. J. Radioanal. Nucl. Chem. 2004, 262, 3542.

(7) Parry, S. J. Radiochim. Acta 2012, 100, 495-501.

(8) ANDRA, National Radioactive Waste Management Agency, In ACO.SP.ASRE.99.001D and ACO.SP.ASRE.99.0002D ANDRA specifications, 2014.

(9) Kupiec, K.; Konieczka, P.; Namieśnik, J. Crit. Rev. Anal. Chem. 2009, 39, 311-322.

(10) Hou, X.; Roos, P. Anal. Chim. Acta 2008, 608, 105-139.

(11) Warwick, P. E.; Kim, D.; Croudace, I. W.; Oh, J. Anal. Chim. Acta 2010, 676, 93-102.

(12) Keith-Roach, M. J. Sci. Total Environ. 2008, 396, 1-11.

(13) Sillanpaa, M.; Sihvonen, M. L. Talanta 1997, 44, 1487-1497.

(14) Patzay, G.; Ramadan, Y.; Tonko, C. J. Radioanal. Nucl. Chem. 2014, 302, 161-168.

(15) Keith-Roach, M. J. Anal. Chim. Acta 2010, 678, 140-148.

(16) Dodi, A.; Monnier, A. J. Chromatogr. A 2004, 1032, 87-92.

(17) De Maquillé, L.; Renaudin, L.; Goutelard, F.; Vial, J.; Jardy, A.; Thiébaut, D. J. Chromatogr. A 2013, 1276, 20-25.

(18) Fritz, J. S.; Gjerde, D. T. In Ion Chromatography, Wiley-VCH, 2009.

(19) Haddad, P. R.; Nesterenko, P. N.; Buchberger, W. J. Chromatogr. A 2008, 1184, 456-473.

(20) Michalski, R. Crit. Rev. Anal. Chem. 2006, 36, 107-127.

(21) Hodge, E. M. J. Chromatogr. Sci., 2000, 38, 353-356. (22) Wahl, K. L.; Wahl, J. H.; Bolz, C. D. LCGC Europe 2010, 23, 188-189.

(22) Wahl, K. L.; Wahl, J. H.; Bolz, C. D. LCGC Europe 2010, 23, 188-189.

(23) Meyer, V. R. In Practical High-Performance Liquid Chromatography, J. Wiley, 2010.

(24) Wund, P.; Gautier, C.; Vial, J.; Thiebaut, D.; Goutelard, F., presented at SEP 2015 conference, Paris, France, March 31-April 2, 2015.

(25) Smith, R. M.; Martell, A. E. In Critical Stability Constants, Plenum Press, 1973.

(26) Gautier, C.; Rey, C.; Roussignol, D.; Machon, E.; Randon, J.; Crozet, M.; Rivier, C., in preparation.

(27) Analysis method establishment Committee, In http://fr.amiando.com/eventResources/O/H/QqXVzYtB2ddd0c/Present ation_CETAMA_2015.pdf, Assessed 20 March 2015.

(28) Wolff, J. C.; Ornemark, U.; Taylor, P. D. P.; De Bievre, P. Talanta 1998, 46, 1031-1040.

(29) Roman, M.; Dovi, R.; Yoder, R.; Dias, F.; Warden, B. J. Chromatogr., 1991, 546, 341-346.

\section{AUTHOR INFORMATION}


